\title{
Aa. Vv., «Le moyen français» 57-58
}

\section{Maria Colombo Timelli}

\section{(2) OpenEdition}

\section{Journals}

\section{Édition électronique}

URL : http://journals.openedition.org/studifrancesi/27253

DOI : 10.4000/studifrancesi.27253

ISSN : 2427-5856

\section{Éditeur}

Rosenberg \& Sellier

\section{Édition imprimée}

Date de publication : 31 décembre 2006

Pagination : $578-580$

ISSN : 0039-2944

\section{Référence électronique}

Maria Colombo Timelli, «Aa. VV., «Le moyen français» 57-58 », Studi Francesi [En ligne], 150 (L | III) | 2006, mis en ligne le 30 novembre 2015, consulté le 08 novembre 2020. URL : http://

journals.openedition.org/studifrancesi/27253; DOI : https://doi.org/10.4000/studifrancesi.27253

Ce document a été généré automatiquement le 8 novembre 2020.

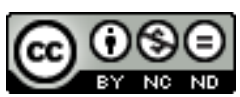

Studi Francesi è distribuita con Licenza Creative Commons Attribuzione - Non commerciale - Non opere derivate 4.0 Internazionale. 


\title{
Aa. Vv., «Le moyen français» 57-58
}

\author{
Maria Colombo Timelli
}

\section{RÉFÉRENCE}

«Le moyen français» 57-58, 2005-2006.

1 Sont réunis dans ce volume les actes du $1^{\text {er }}$ Colloque international du Groupe de recherche sur le moyen français, qui s'est déroulé à l'Université catholique de Louvain (Louvain-la-Neuve) en mai 2003, publiés par Claude Thiry et Tania Van Hemelryck. Le sujet choisi, «La littérature à la cour de Bourgogne, actualités et perspectives de recherche», a stimulé la présentation de travaux en cours ainsi que la réflexion sur les méthodes et sur les recherches encore à entreprendre. Les notices qui suivent ont été regroupées autour de quelques axes significatifs: la Grande Rhétorique, la production historiographique, la littérature en prose (romans, compilations, nouvelles), le théâtre. Deux communications, respectivement de Giuseppe Di Stefano et de Céline Van Hoorebeeck, se situent à part.

2 Annelies blöm, 'Si jeunesse savait, si vieillesse pouvait': une analyse de la personnalité poétique de Michault Taillevent dans son 'Passe Temps', pp. 11-25. La présence constante de la première personne dans le Passe-Temps cache deux 'je' différents: le 'je' d'autrefois, intra-diégétique, chargé de toutes les fautes de la jeunesse, et le 'je' du poète narrateur, extra-diégétique, auteur d'un poème moralisant. Dans les deux cas, selon A. Bloem, la connivence du lecteur demeure possible, en raison du haut niveau de généralisation et, en contrepartie, du faible degré de subjectivité adoptés par Michault Taillevent.

Laurent BOZARD, Le poète et la princesse. Jean Molinet, Jean Lemaire de Belges, Jean Marot et leurs 'muses': Marguerite d'Autriche et Anne de Bretagne, pp. 27-40. L'auteur étudie les liens qui unissent les trois poètes et les deux princesses, ainsi que l'expression poétique de cet attachement. Sans être sa muse, Marguerite d'Autriche demeure pour Jean Molinet l'emblème de la paix. Lemaire, poète mais aussi collaborateur de Marguerite, passe en 1511 à la cour de France et à Anne de Bretagne: ses vers s'adaptent alors aux circonstances et aux nouvelles destinataires. En revanche, la reine de France est la 
véritable muse de Jean Marot, quasi entièrement voué à sa cause de 1506 à 1514, date de la mort d'Anne.

4 David cowling, L'emploi de la métaphore dans les textes bourguignons du XVe siècle: bilan et perspectives de recherche, pp. 55-66. À partir d'une approche cognitiviste de la métaphore, qui met en rapport un 'domaine-source' (ici, la maison) et un 'domainecible' (l'État), David Cowling montre l'apport des indiciaires de Philippe le Bon et de Charles le Téméraire à une histoire de la métaphore du bâtiment appliquée à l'État au $\mathrm{XV}^{\mathrm{e}}$ siècle. Si Chastelain se révèle encore proche de la vision négative des historiographes français, chez lesquels la 'maison' est surtout marquée par la fragilité au risque de l'effondrement et de la ruine, avec Molinet et surtout Lemaire de Belges le 'bâtiment' assume de plus en plus les caractères d'une construction dont la perfection et la permanence sont indiscutables. La métaphore joue ainsi un rôle fondamental dans l'élaboration, peut-être en partie inconsciente, d'une vision précise de l'État bourguignon à la fin du Moyen Âge.

5 Estelle DOUDET, La personnalité poétique à l'aube de la Renaissance. George Chastelain et la filiation littéraire chez les Grands Rhétoriqueurs, pp. 105-121. Après s'être forgé pour luimême une fausse généalogie glorieuse, George Chastelain devient - avec Alain Chartier - le maître célébré par au moins deux générations de Grands Rhétoriqueurs. À travers le dialogue poétique et épistolaire et l'élaboration de textes à plusieurs mains, se tisse alors la sodalitas d'un groupe qui reconnaît l'autorité du grand indiciaire, tout en affirmant la liberté de création et l'originalité de chacun. En ce sens, selon E. Doudet, la Grande Rhétorique marque le passage du Moyen Âge à la poésie de la Renaissance.

6 Martial MARTIN, Le prosimètre des rhetoriqueurs bourguignons: miroir d'une cour en crise, pp. 249-262. La riche production de prosimètres par Chastelain, Molinet et Lemaire de Belges se fonde sur des structures relativement stables, des cadres peu variés, des personnages divers mais des personnifications et des allégories récurrentes. Ce qui fait l'originalité d'un tel ensemble, au-delà de la diversité des réalisations, c'est alors l'émergence d'un discours politique.

7 Virginie MINET-MAHY, Les systèmes de représentation de l'auteur et du lecteur dans le texte allégorique: figures idéales et vocations poétiques, pp. 263-282. Le fonctionnement de certaines métaphores et, plus en général, du discours allégorique dans la poésie de $\mathrm{G}$. Chastelain et de J. Molinet confirme la thèse de V. Minet-Mahy d'une profonde intégration de l'auteur et du lecteur dans l'espace fictionnel des Grands Rhétoriqueurs. Si l'auteur de la fin du Moyen Âge est de plus en plus persuadé de jouer un rôle politique dans le débat public et propose une écriture désormais engagée dans le monde, le lecteur se trouve directement impliqué dans le texte par les appels directs à sa propre mémoire littéraire, ainsi qu'à des images culturelles et à des valeurs sociales communes.

8 Anne schoysman (Jean Lemaire de Belges et la Généalogie d'Anne de la Tour d'Auvergne dans le ms. 74 G 11 de La Haye (1518), pp. 315-333). A. Schoysman étudie dans les détails un manuscrit non daté, mais datable 1518, dont le contenu doit être attribué à Jean Lemaire de Belges. Il s'agit d'une compilation de plusieurs textes, dont un roman des origines en prose, deux généalogies de la famille de La Tour d'Auvergne, et des pièces hétéroclites rapportées aux possessions de la même famille. Exemplaire de dédicace d'un manuscrit de nature privée, certainement non autographe, il confirmerait que Lemaire, sans doute infirme, était encore actif en 1518, et obligerait à reculer la date de sa mort vers 1524. 
Susie SPEAKMAN SUTCH, La réception du 'Chevalier délibéré' d'Olivier de la Marche aux XVe et XVIe siècles, pp. 335-350. L'analyse des manuscrits et imprimés français du Chevalier deliberé parus entre la fin du XVe siècle et 1540 permet de mieux cerner les milieux dans lesquels ce poème a circulé. La présence d'armoiries et devises sur les manuscrits, ainsi que la richesse de l'apparat iconographique révèlent des destinataires / commanditaires / possesseurs appartenant à la plus haute aristocratie. Deux imprimés sur vélin, aux gravures peintes, circulèrent dans le même milieu, alors que d'autres éditions plus tardives étaient manifestement destinées à un public plus vaste et moins exigeant.

10 Björn-Olav Dozo, Représentations de la figure de Charles Quint: des espoirs suscités aux traces dans une chronique française de la fin de sa vie. Comparaison de trois représentations: Molinet, Ladam, Le Blond, pp. 123-138. Les images de Charles Quint qui ressortent de l'œuvre de trois chroniqueurs excentrés - sur le plan chronologique ou géographique - sont plus variées que celles que l'empereur a pu influencer directement. Jean Molinet souligne surtout ses liens avec la dynastie bourguignonne et les espérances de paix suscitées par sa naissance; Nicaise Ladam, tout en insistant sur sa jeunesse et son apparente vulnérabilité, fait de Charles l'espoir politique de l'Europe; le portrait esquissé par Jean Le Blond enfin montre un prince autoritaire œuvrant plus pour son propre pouvoir que pour l'unité de la chrétienté.

11 Sylvie LEFÈVRE, Une tradition bourguignonne d'un dialogue d'humanistes: la biographie anecdotique d'Alphonse $V$ d'Aragon, pp. 227-247. Le De dictis et factis Alphonsi regis d'Antoine Beccadelli dit Panormita, portrait idéalisé d'un roi parfait puisque philosophe, fut achevé en 1455, puis complété par des additions d'Aeneas Sylvius Piccolomini; le texte entier fut traduit en français entre 1469 et 1476 par Jean Lorfèvre, grand notable de la cour de Bourgogne. Le répertoire de manuscrits et éditions anciennes établi par $\mathrm{S}$. Lefèvre témoigne d'une diffusion complexe de la rédaction latine (les textes de Beccadelli et de Piccolomini ayant circulé aussi bien séparés que réunis), alors que la traduction française n'est connue que par un manuscrit aujourd'hui conservé à Cracovie.

12 Peter NOBLE, Commynes et Charles le Téméraire, pp. 283-290. Le caractère partial des Mémoires de Commynes à l'égard de sa fuite de la cour de Bourgogne pour passer au service de Louis XI a été reconnu depuis longtemps par la critique. P. Noble attribue le silence de l'indiciaire non pas à un sentiment de culpabilité (interprétation avancée par Jean Dufournet), mais plutôt à un désir de justification, qui aurait amené Commynes à noircir le portrait de Charles le Téméraire en se concentrant sur les défauts de son caractère.

13 Maria colombo timell, Pour une nouvelle édition critique de 'La Manequine' en prose de Jean Wauquelin: quelques réflexions préliminaires, pp. 41-54. Malgré les mérites incontestables de l'édition fournie par Hermann Suchier en 1884, une nouvelle édition critique de La Manequine en prose doit aujourd'hui être envisagée, aussi bien pour des raisons littéraires (une propre dignité est désormais reconnue aux 'mises en prose'; l'auteur bourguignon est par ailleurs mieux connu) que linguistiques et philologiques (l'édition Suchier ne contient ni analyse linguistique ni glossaire; le manuscrit n'est que sommairement décrit). M. Colombo Timelli offre ici une réflexion sur le rapport entre certaines leçons du manuscrit unique de Turin (Biblioteca Nazionale e Universitaria, L.IV.5) et les passages correspondants dans le seul manuscrit conservé du roman en vers de Philippe de Remi (BnF, fr. 1588). 

convergence des intrigues amoureuses et politiques dans le 'Cligés' en prose, pp. 67-86. Analyse thématique du Cligés en prose (1454), centrée sur l'amour adultère de Cligés et Fenice: leur liaison est entièrement justifiée par le prosateur au nom de la trahison opérée par Alix, 'mauvais chef', faux empereur de Constantinople, auquel il est légitime de s'opposer. Par ailleurs, l'amour des deux protagonistes se trouve ultérieurement soutenu par une personnification supra-naturelle, Amour, qui leur assure un secours inconditionnel et toujours précieux. C. Deschepper conteste enfin, à juste titre, pour cette 'prose' l'étiquette de 'miroir' offert aux Ducs: l'auteur du XV siècle a plutôt tenté d'intégrer l'univers arthurien et l'image d'Alexandre empereur de Constantinople aux préoccupations politiques de son milieu.

Jean DevauX, L'art de la mise en prose à la cour de Bourgogne: Jean Molinet, dérimeur du 'Roman de la Rose', pp. 87-104. La prosification de Jean Molinet se signalant par son extrême fidélité au roman source, elle permet des observations de grand intérêt quant à l'évolution de la langue du XIII ${ }^{e}$ au XV $\mathrm{XV}^{\mathrm{e}}$ siècle. Jean Devaux analyse de près les cinq premiers chapitres, correspondant aux 1300 premiers vers du poème. Sur le plan de la morphologie, la modernisation paraît constante, ainsi que pour la syntaxe, où l'imbrication s'affirme comme le procédé marquant du texte en prose; il est en revanche plus difficile de classer les modifications lexicales, et surtout d'en expliquer les motivations. Par rapport à d'autres proses 'bourguignonnes' le Roman de la Rose de Molinet se révèle donc plus fidèle au modèle, et proche de l'Ovide moralisé et du Livre de Regnart pour la séparation nette entre récriture et 'moralisation'.

Philippe FRIEDEN, La Rose et le Christ: lecture eucharistique du 'Romant de la Rose moralisé', pp. 139-151. Cette lecture du Romant de la Rose moralisé est fondée sur la présence du Christ dans 32 moralités, et notamment dans les moralités accompagnant l'épisode de la Fontaine de Narcisse (Guillaume de Lorris) et celui de la Fontaine du Parc (Jean de Meun); le dernier chapitre en particulier révèle définitivement la relecture opérée par Molinet, avec l'identification du Christ et de la Rose.

17 Sandrine hÉRICHÉ-PRADEAU ('Les Faicts et Conquestes d'Alexandre' de Jehan Wauquelin: un espace de réécritures variées, pp. 153-175) montre la variété des procédés de réécriture appliqués par Wauquelin dans sa vaste compilation. Le prosateur utilise ses deux sources principales (les deux premières branches du roman en alexandrins et une traduction française de l'Historia de Preliis) avec une relative fidélité, qui n'exclut ni les réductions / suppressions ni certaines amplifications. Quant aux sources secondaires, Wauquelin procède plutôt par extraction de blocs narratifs, auxquels il reste plus ou moins fidèle. Loin d'être le résultat d'un simple assemblage de textes, la compilation du $\mathrm{XV}^{\mathrm{e}}$ siècle offre un nouveau roman d'Alexandre, inédit et cohérent.

18 Frédérique JOHAN, Quelques accents uniques pour une autre lecture du 'Petit Jehan de Saintré: l'apport des images du Bruxellensis 9547, pp. 177-193. Il s'agit d'une analyse partielle des illustrations (une vingtaine sur 77) qui ornent le ms. de Bruxelles, mises en rapport avec les titres de chapitre paraissant dans le ms. Paris BnF naf 20234. F. Johan essaie de prouver que, surtout dans la dernière partie du roman, l'artiste imposerait sa propre interprétation de la morale proposée par Antoine de La Sale.

Giovanni Palumbo, La 'Chanson de Roland' dans les 'Croniques et conquestes de Charlemaine': le problème des sources, pp. 291-314. À la recherche des sources majeures de la compilation de David Aubert, G. Palumbo montre que les Croniques combinent les deux traditions, turpinienne et rolandienne, et surtout il précise - grâce à des sondages significatifs -

Studi Francesi, 150 (L | III) | 2006 
les versions auxquelles Aubert a puisé: une traduction française de Turpin attribuée à 'maistre Jehan', et une version du Roland rimé peut-être déjà mise en prose; à ces remaniements s'ajoutent deux blocs narratifs extérieurs: la prise de Narbonne et l'histoire de Galien.

20 Tania VAN HEMELRYCK, Soumettre le 'Perceforest' à la question. Une entreprise périlleuse?, pp. 367-379. La constatation de la datation tardive de tous les manuscrits de Perceforest amène $\mathrm{T}$. Van Hemelryck à lancer une nouvelle hypothèse pour la date de rédaction du texte. Quelques éléments internes et externes à l'œuvre pourraient en effet etayer l'hypothèse d'une composition 'bourguignonne' du début du $\mathrm{XV}^{\mathrm{e}}$ siècle.

21 Nelly LABÈRE, Regarder par le trou de la lorgnette: 'L'assez apparente verité' des 'Cent Nouvelles Nouvelles', pp. 203-226. Les CNN utilisent le motif traditionnel du 'regard' en en faisant le centre thématique du recueil: la vue et ses défauts constituent en effet le noyau de plusieurs contes, peuplés de myopes, presbytes, borgnes (réels et métaphoriques). Qui plus est, la vue, le trou, la béance, exploités grossièrement, ne sont pourtant pas sans interroger le lecteur sur le rapport entre signe et sens, entre vérité et apparence.

Cynthia SYOEN, Un 'merveilleux' quotidien: l'amour contrarié dans les 'Cent Nouvelles Nouvelles' , pp. 351-365. La réflexion sur l'amour et sur la place des femmes est bien au centre de nombreuses nouvelles du recueil. C. Syoen étudie le rapport entre cette réflexion d'une part et, de l'autre, le discours médical et les croyances populaires, qui rejoignent la vision chrétienne et surtout mysogine du Moyen Âge. Dans les $C N N$ la femme se révèle en même temps castratrice et sujette à l'homme: le rire seul saura assumer un rôle rassurant, en même temps libérateur et exorcisateur.

Tamás KARÁTH, La tradition bourguignonne des représentations de la Passion à la fin du Moyen Âge. La Passion de Biard: un témoignage d'une dévotion personnelle, pp. 195-201. Le ms. copié par Philippe de Biard dans la seconde moitié du XVe siècle (Paris BnF, naf 4085) suit un principe de composition proche de celui des livres d'heures. Le format, la graphie, indiqueraient aussi un usage privé; mais surtout la réécriture, tout en prouvant la circulation du mystère dans les milieux bourguignons à une époque où les représentations dramatiques de la Passion semblent avoir disparu, transforme le texte théâtral en un instrument de réflexion individuelle sur la foi.

Céline VAN HOOREBEECK, 'Item, ung petit livre en franchois...' La littérature française dans les librairies des fonctionnaires des ducs de Bourgogne, pp. 381-413. Sur la base des relevés écrits d'une quinzaine de 'librairies' de fonctionnaires bourguignons décédés entre 1444 et 1543, C. Van Hoorebeeck essaie de vérifier dans quelle mesure ces bibliothèques reflètent les goûts de la cour ducale et de la haute aristocratie. En s'appuyant sur une série de paramètres significatifs (la langue des ouvrages, la typologie des œuvres, la qualité et la valeur des livres, manuscrits et imprimés, le contenu), elle peut conclure sur une relative autonomie de ces collections, qui constituaient d'abord un outil de travail pour des fonctionnaires qui ne renonçaient cependant pas entièrement à trouver dans la lecture un espace de divertissement.

Giuseppe DI STEFANO, L'acception libre: relire Jean Molinet, pp. 415-430. La parution récente du Dictionnaire érotique de Rose M. Bidler (2002) permet à G. Di Stefano de relire les Pronostications joyeuses de Molinet (éd. Koopmans - Verhuyck 1998, TLF 496) et d'y glaner un nombre importants de lexèmes et locutions à double sens. Prudence impose pourtant de se demander toujours si l'auteur a consciemment manipulé les mots ou bien si l'interprétation érotique est simplement l'effet inévitable de la polysémie. 